\title{
$\mathrm{TBC}$ 에서 부채널공격을 고려한 효율적인 지수 연산
}

\author{
박 영 호, ${ }^{\dagger}$ 장 남 수 $^{\ddagger}$ \\ 세종사이버대학교
}

\section{An efficient exponentiation method against side channel attacks in Torus-Based-Cryptosystem*}

\author{
Young-Ho Park, ${ }^{\dagger}$ Nam Su Chang ${ }^{\ddagger}$
}

Sejong Cyber University

\begin{abstract}
요 약
본 논문은 Torus-Based-Cryptosystem 중 $T_{2}(p)$ 에서 부채널공격을 방지하는 효율적인 지수 연산방법을 제안 한다. 제안한 지수 연산방법은 일반적인 지수 연산보다 더 효율적일 뿐만 아니라 제곱과 곱셈 연산의 계산량 차이를 없게하여 SPA 공격에 안전하다. 또한 상군(quotient group)의 특성을 이용하여 지수 연산시 메시지를 난수화하여 제1차 DPA 공격도 방어할 수 있다.
\end{abstract}

\section{ABSTRACT}

We propose an efficient exponentiation method which is resistant against some side channel attacks in $T_{2}(p)$, Torus-Based-Cryptosystem. It is more efficient than the general exponentiation method in $T_{2}(p)$ and is resistant against SPA by using that the difference of squaring and multiplication costs is negligible. Moreover, we can randomize a message in exponentiation step using the characteristic of quotient group which naturally protects against the first DPA.

Keywords: Torus-Based-Cryptosystem, Quotient Group, Side Channel Attack, SPA, DPA, Finite Field, Public Key Cryptosystem

\section{I. 서 론}

엘가말(ElGamal) 암호시스템[1]과 DSA[2]와 같이 유한체 곱셈군에서 이산대수문제를 이용한 많은 시스템들이 암호학적 응용으로 유용하게 제안되었다. 이들은 소수체(prime field)의 부분군을 사용하며, 이 시스템의 안전성은 큰 소수를 위수로 갖는 부분군 에서의 이산대수문제는 유한체 전체 곱셈군의 이산대

접수일(2013년 5월 22일), 게재확정일(2013년 5월 29일)

* 본 연구는 2010 년도 정부(교육과학기술부)의 재원으로 한국연구재단의 지원을 받아 수행된 기초연구사업입니다. (No. 2010-0011511).

† 주저자, youngho@sjcu.ac.kr

\$ 교신저자, nschang@sjcu.ac.kr(Corresponding author)
수문제만큼 어렵다는데 근거를 두고 있다[3]. 또한 소수체 대신에 확장체의 부분군을 사용하는 시스템들 이 주목을 받고 있으며 $\mathrm{LUC}[4]$ 와 XTR[9]이 공개 키 암호법으로 소개되었다. 특히 2003년 Park외 2 명〔6]은 이차확창체의 상군에서의 이산대수문제를 고 려한 암호시스템을 제안하였으며 이와 독립적으로 Rabin와 Silverberg[7]가 Torus based Cryptography(TBC)을 제안하였고 그 후 많은 연 구가 진행되고 있다[8-13].

$\mathrm{TBC}$ 는 공개키 파라메터의 크기를 줄여 통신량을 절약할 뿐만 아니라 효율적인 연산방법들이 가능하다. $\mathrm{LUC}$ 와 XTR과 다르게 기존의 유한체의 부분군 이산 대수 시스템에서의 스킴들을 사용할 수 있으며 따라서 기존의 ElGamal 암호시스템, DSA, DH 키교환 스 
킴들을 이용한 모든 프로토콜들에 큰 수정 없이 그대 로 적용 가능하다. 또한 윈도우방법과 선계산방법을 사용하여 효율적인 지수 연산 뿐만 아니라 다승연산 (multi-exponentiation)을 수행할 수 있는 장점도 있다.

한편 최근 스마트카드, RFID, NFC, 센서네트워 크 등 스마트 기기들이 많이 사용되고 있으며 이러한 기기들에 대한 부채널공격(Side Channel Attack) 〔14〕의 위협이 날로 증가하고 있다. XTR와 같은 sequence based 암호의 경우도 다양한 부채널 공격 에 취약하다는 것이 발표되었다[15-17]. 따라서 Torus 기반의 암호 $(\mathrm{TBC})$ 에서도 부채널공격을 막는 효율적인 연산의 연구가 필요한 실정이다.

본 논문에서는 상군의 특성을 이용하여 $\mathrm{TBC}$ 중 $T_{2}(p)$ 에서 부채널공격을 막는 효율적인 알고리즘을 제안한다. SPA(Simple Power Analysis)를 막는 근본적인 방법 중 하나인 제곱연산과 곱셉연산의 계산 량의 차이를 없게(negligible) 만들어 일반적인 SPA 대응 방법인 Square-and-MultiplicationAlways Method(SMAM)과 Dummy Operations Method(DOM)와 달리 추가적인 연산량이 들지 않는 SPA 대응 연산방법을 제안한다. 또한 상군 의 특성상 지수 연산에서도 자연스럽게 메시지를 난수 화 하므로 제 1 차 DPA 공격에도 추가적인 연산량 없 이 효율적으로 방어할 수 있음을 보인다.

논문의 구성은 다음과 같다. 2절에서 상군과 상군 에서의 연산에 대해 설명하고 3절에서 $\mathrm{SPA}$ 를 막는 효율적인 알고리즘을 소개하고 4절에서 결론을 맺는 다.

\section{II. 상군(Quotient Group)과 상군에서의 연산}

\section{1 상군(Quotient Group)}

양의 정수 $m$ 와 소수 $p$ 에 대해 $l=p^{m}$ 라 놓자. $F_{l}$ 를 $l$ 개의 원소를 갖는 유한체라 하고, $F_{l}(\omega)$ 를 $F_{l}$ 에서 이차 기약다항식의 근 $\omega$ 로 확장한 유한체라 하자. 이 때 $F_{l}(\omega)$ 는 $F_{l}$ 위에서 차수 2 인 벡터공간으로 $\{1, \omega\}$ 는 기저(basis)가 된다. 따라서 $F_{l}(\omega)$ 의 모든 원소는 다 음과 같은 일차결합으로 표현된다:

$$
\alpha=x+y \omega, \quad x, y \in F_{l} .
$$

$F_{l}(\omega)^{*}$ 는 위수가 $l^{2}-1$ 인 곱셈군으로 부분군 $F_{l}^{*}$ 를 포함하며 $\left|F_{l}(\omega)^{*} / F_{l}^{*}\right|=l+1$ 이다. $F_{l}(\omega)^{*}$ 의 곱으로 유
도된 연산을 갖는 $F_{l}^{*}$ 의 상군(quotient group)을 $G:=F_{l}(\omega)^{*} / F_{l}^{*}$ 라 하자. 더 명확하게, $\alpha \in F_{l}(\omega)^{*}$ 를 포 함하는 류(class)를 $[\alpha]$ 라 표시하자. 여기서, $[\alpha]=[\beta] \in G \Leftrightarrow \alpha \beta^{-1} \in F_{l}^{*}$ 임을 알 수 있다. 그리고 $G$ 에서 연산을 $[\alpha][\beta]=[\alpha \beta]$ 로 정의한다. 자세한 내용은 [6]을 참조 바란다.

정리 1.

(1) $G$ 는 $F_{l}(\omega)^{*}$ 의 곱으로 유도된 연산을 갖는 위수 가 $l+1$ 인 순환군이다.

(2) 만일 $\alpha \in F_{l}(\omega)^{*}$ 이고 $\alpha \not f F_{l}$ 이면 유일한 원소 $g \in F_{l}$ 가 존재하여 $[\alpha]=[g+\omega]$ 이다.

위 정리 1 로부터 일대일 대응함수 $\phi: G(1] \rightarrow F_{l}$ 를 정의할 수 있다.

$$
\phi\left(\left[a_{0}+a_{1} \omega\right]\right)=a_{0} a_{1}^{-1} .
$$

또한 $\phi([1])=i d$ 로 정의하면 함수 $\phi$ 는 $G$ 의 모든 원소로 확장된다. 따라서 함수 $\phi$ 는 상군의 모든 원소 를 $F_{l} \cup i d$ 로 표현할 수 있다.

\section{2 소수체의 이차확장체에서의 연산}

상군 $G=F_{l}(\omega) * / F_{l}^{*}$ 에서의 이산대수문제를 기 반한 암호는 정확히 $\mathrm{TBC}$ 중 $T_{2}(l)$ 에 해당된다. $T_{2}(l)$ 를 사용한 다양한 암호시스템들은 모두 $F_{l}(\omega)$ *에 있 는 임의의 원소 $\mathrm{a}$ 와 임의의 양의정수 $n$ 에 대한 지수 연산 $[\alpha]^{n}$ 을 사용하며 이 연산이 암호시스템의 성능을 좌우하는 가장 중요한 부분이다. 상군의 특성상 $[\alpha]^{n}=\left[\alpha^{n}\right]$ 이기 때문에, 상군 $G$ 에 있는 원소의 지수 연산은 $F_{l}(\omega)$ 에 있는 원소의 지수 연산으로 쉽게 변형 하여 계산할 수 있다.

본 논문에서는 편의를 위해 $l=p$ 이고 소수 $p \equiv 2(\bmod 3)$ 인 $T_{2}(p)$ 의 경우만을 다룬다. 만일 $F_{p}(\omega)$ 의 원소를 표현하기 위해 $F_{p}$ 위의 이차 기약다항식 $X^{2}+X+1$ 과 다항식기저(polynomial basis)를 사 용하면 다음과 같은 보조정리를 얻을 수 있다 (〔18,19] 참조). 본 논문에서 연산량을 측정하기 위 해 [18〕에서 사용한 것처럼 $F_{p}$ 에서의 연산 기호 $\mathrm{M}, \mathrm{S}, \mathrm{D}$ 를 사용하는데 $\mathrm{M}$ 과 $\mathrm{S}$ 는 모듈러 감산을 사용 하지 않는 두 개의 정수의 곱셈과 제곱을 각각 의미하 며, $\mathrm{D}$ 는 모듈러 감산 연산을 의미한다. $F_{p}$ 에서의 곱 
셈연산은 $\mathrm{M}+\mathrm{D}$ 연산으로 계산된다. 따라서 일반적인 지수 연산의 계산모델을 사용하여 $F_{p}$ 에서의 덧셈과 뺄셈 연산은 무시하며 지수 연산의 계산량 측정단위로 $\mathrm{M}=\mathrm{D}=0.5$ 와 $\mathrm{S}=0.3$ 을 사용한다[18].

보조정리 1. 소수 $p \equiv 2(\bmod 3)$ 인 $F_{p}(\omega)$ 의 원소 $\alpha, \beta$ 는 다음을 만족한다.

(1) $\alpha^{p}$ 계산량은 거의 없다.

(2) $\alpha^{2}$ 계산량은 $2 \mathrm{M}+2 \mathrm{D}$ 이다.

(3) $\alpha \beta$ 계산량은 $3 \mathrm{M}+2 \mathrm{D}$ 이다.

또한 $[\alpha] \in G$ 에서 $[\alpha]^{p+1}=i d$ 이므로 $\quad[\alpha]^{-1}=[\alpha]^{p}$ $=\left[\alpha^{p}\right]$ 이며 역원 $[\alpha]^{-1}$ 은 보조정리 1 에 의해 계산량이 들지 않으므로 signed binary 방법을 사용하여 지수 연산을 효율적으로 계산할 수 있다.

보조정리 2. 소수 $p \equiv 2(\bmod 3)$ 이고 $[\alpha] \in G$,

$$
\alpha=x+y \omega, \phi[\alpha]=g \in F_{p} \text { 이면 }
$$

(1) $[x+y \omega]^{-1}=[(x-y)-y \omega]$.

(2) $\phi\left([\alpha]^{-1}\right)=1-g \in F_{p}$.

증명.

(1) $(x+y \omega)((x-y)-y \omega)=x^{2}-x y+y^{2} \in F_{p}$

(2) $\phi\left([\alpha]^{-1}\right)=\phi([(x-y)-y \omega])$

$$
\begin{aligned}
& =\phi\left(\left[-y\left(\left(-x y^{-1}+1\right)+\omega\right)\right]\right) \\
& =\phi\left(\left[\left(-x y^{-1}+1\right)+\omega\right]\right)=-x y^{-1}+1 \\
& =1-\phi[\alpha]=1-g .
\end{aligned}
$$

상군 $G=F_{p}(\omega)^{*} / F_{p}^{*}$ 에서 $k$ 비트를 갖는 지수 $n$ 에 대하여 $[\alpha]^{n}$ 의 효율적인 연산을 위해 signed binary 방법과 NAF window 방법을 사용할 수 있 다([11] 참조). 일반적인 left-to-right binary method 의 running time은 $k / 2$ 곱셈과 $k$ 의 제곱 연산이 소요된다. 반면 윈도우 사이즈 $w$ 의 $\mathrm{NAF}$ 의 running time은 선계산량이 $2^{w-2}-1$ 곱셈과 한 번 의 제곱연산 그리고 $\frac{k}{w+1}$ 곱셈과 $k$ 의 제곱연산이 소 요된다. 따라서 보조정리 1 과 2 에 의해서 다음과 같 은 정리를 얻는다.

정리 2. 소수 $p \equiv 2(\bmod 3), \quad[\alpha] \in G \quad$ 이면 $\left\lceil\log _{2} n\right\rceil=k$ 인 양의 정수 $n$ 에 대하여 $[\alpha]^{n}$ 은
(1) Left-to-right binary method에 의해 $((7 / 2) M+3 D) k$ 연산량이 필요하며 $F_{p}$ 에서의 평균 $3.25 k$ 곱셈으로 계산된다.

(2) 윈도우 사이즈 $w$ 의 NAF에 의해

$\left(3 \cdot 2^{w-2}-1\right) M+2^{w-1} D$

$$
+k\left(\left(\frac{3}{w+1}+2\right) M+\left(\frac{2}{w+1}+2\right) D\right)
$$

연산량이 필요하며 $F_{p}$ 에서의 평균

$$
\left(5 \cdot 2^{w-3}-\frac{1}{2}\right)+k\left(\left(\frac{5}{2(w+1)}+2\right)\right)
$$

곱셈으로 계산된다.

정리 2는 $T_{2}(p)$ 에서의 지수 연산이 같은 보안강도 를 갖는 소수체에서의 원래 지수 연산보다 효율적인 것을 나타낸다. 소수 $\mathrm{p}$ 가 512 비트인 경우 1024 비트 의 소수체에서의 지수 연산보다 $G=F_{p}(\omega)^{*} / F_{p}^{*}$ 에서의 지수 연산이 대략 $40 \%$ 의 속도가 향상됨을 알 수 있다 [6].

\section{III. 부채널 공격을 막는 효율적인 알고리즘}

부채널공격 중 $\mathrm{SPA}$ (Simple Power Analysis) 를 막는 대표적인 방법으로 지수의 비트 값에 상관없 이 항상 곱셈과 제곱연산을 수행하는 SMAM과 추가 적인 계산을 수행하는 $\mathrm{DOM}$ 이 주로 사용된다. 하지 만 이들 방법은 추가적인 계산량의 증가를 가져온다. 본 논문에서는 $\mathrm{SPA}$ 를 막는 근본적인 방법 중 하나인 제곱연산과 곱셈연산의 계산량의 차이를 없게 만들어 (negligible) 추가적인 연산량이 들지 않는 연산방법 을 제안한다. 만일 소수 $p \equiv 2(\bmod 3)$ 이며 $[\alpha] \in G$, $\alpha=x+y w$ 일 때 $[\alpha]=[g+w], g=\phi[\alpha]=x y^{-1}$ 라 하자. 제안된 방법의 주 아이디어는 연산 시 반복적으로 곱 셈에 사용되는 $\alpha=x+y w$ 대신 같은 값을 갖는 $g+w$ 를 사용하여 곱셈 연산량을 감소시키는 것이다. 따라 서 binary NAF 방법을 사용하여 다음과 같은 지수 연산 알고리즘을 제안한다.

[알고리즘 1]에서 $A \cdot(g+w)$ 와 $A \cdot(1-g+w)$ 의 연산량을 계산하면 다음과 같다. 임의의 $A=$ $x+y w \in F_{p}(w)$ 에 대하여

$$
\begin{aligned}
(x+y w)(g+w) & =(x g-y)+(x+y g-y) w \\
(x+y w)(1-g+w) & =(x-x g-y)+(x-y g) w
\end{aligned}
$$

이므로 각각 $2 M+2 D$ 의 연산으로 계산할 수 있다. 
〔알고리즘 1〕 SPA를 막는 효율적인 지수 연산

Input : $[\alpha]=[g+w]$, 양의 정수 $n$

Output: $[\alpha]^{n}$

1. $N A F(n)=\sum_{i=0}^{k-1} d_{i} 2^{i}, d_{i} \in\{-1,0,1\}$ 를

계산한다.

2. $A \longleftarrow 1$

3. For $i$ from $k-1$ downto 0 do

$3.1 A \leftarrow A^{2}$

3.2 If $d_{i}=1$ then $A \leftarrow A \cdot(g+w)$

3.3 If $d_{i}=-1$ then $A \leftarrow A \cdot(1-g+w)$

4. Return : $[A]$

그러므로 [알고리즘 1)의 3.1 단계의 제곱연산과 3.2 와 3.3 단계의 연산 모두 $2 M+2 D$ 으로 동일한 연 산량으로 계산되며 두 연산량의 차이는 없게 된다. 따 라서 위 제안된 알고리즘은 추가연산 없이 SPA 공격 을 막는 효율적인 알고리즘일 뿐만 아니라 $F_{p}(\omega)$ 의 일 반적인 지수 연산을 사용하는 것보다 더 효율적임을 알 수 있다. 또한 위 제안된 알고리즘은 선계산을 이 용한 윈도우 사이즈 $w$ 의 NAF 알고리즘으로 쉽게 변 형할 수 있다. ‘표 1)은 기존 알고리즘과 제안 알고리 즘의 지수 연산에 대한 연산량을 비교한 것이다.

〔표 1〕에서 SMAM 이나 DOM 는 지수의 비트 값에 상관없이 동일하게 곱셈과 제곱연산이 수행되어 야 하므로 NAF 방법이 효율적이지 않으므로 binary 지수 연산 방법을 사용하는 것이 효율적이다. 일반적 으로 $w-\mathrm{NAF}$ 알고리즘을 사용할 때 가장 효율적인 윈도우 사이즈는 $\left\lceil\log _{2} n\right\rceil=k$ 의 크기에 따라 다르다. 제안된 알고리즘과 일반적인 $w-\mathrm{NAF}$ 알고리즘 모두 $k=160,224$ 또는 256 일 경우 $w=5$ 인 NAF 방법 이 가장 효율적이다. 따라서 〔표 2〕에서는 $w=5$ 인 $\mathrm{NAF}$ 방법을 사용하였을 때 기존의 알고리즘과 제안

〔표 1) 지수 연산량 비교

\begin{tabular}{|c|c|c|}
\hline & $F_{p}$ 에서의 평균 곱셈량 & 방법 \\
\hline $\begin{array}{c}\text { 일반적인 } \\
\text { 지수 연산 } \\
\text { 알고리즘 }\end{array}$ & $\begin{array}{c}\left(5 \cdot 2^{w-3}-(1 / 2)\right) \\
+k((5 /(2(w+1))+2))\end{array}$ & $\mathrm{w}-\mathrm{NAF}$ \\
\hline $\begin{array}{c}\mathrm{SMAM} \\
(\mathrm{DOM})\end{array}$ & $4.5 \mathrm{k}$ & $\begin{array}{c}\text { binary } \\
\mathrm{SMAM}\end{array}$ \\
\hline $\begin{array}{c}\text { 제안된 } \\
\text { 알고리즘 }\end{array}$ & $2^{w-1}+k\left(\frac{2}{(w+1)}+2\right)$ & $\mathrm{w}-\mathrm{NAF}$ \\
\hline
\end{tabular}

〔표 2〕 $w=5$ 인 NAF 방법을 사용한 지수 연산량 비교

\begin{tabular}{|c|c|c|}
\hline & $F_{p}$ 에서의 평균 곱셈량 & 방법 \\
\hline $\begin{array}{c}\text { 일반적인 지수 } \\
\text { 연산 알고리즘 }\end{array}$ & $2.4 k+19.5$ & $5-\mathrm{NAF}$ \\
\hline $\begin{array}{c}\mathrm{SMAM} \\
(\mathrm{DOM})\end{array}$ & $4.5 \mathrm{k}$ & $\begin{array}{c}\text { binary } \\
\mathrm{SMAM}\end{array}$ \\
\hline 제안된 알고리즘 & $2.3 k+16$ & $5-\mathrm{NAF}$ \\
\hline
\end{tabular}

된 알고리즘의 효율성을 비교한 것이다.

또한 상군 $\mathrm{G}$ 의 원소 $[\alpha],[\beta] \in G$ 에 대하여 $[\alpha]=$ $[\beta] \Leftrightarrow \alpha \beta^{-1} \in F_{p}^{*}$ 이므로 임의의 0이 아닌 난수 $r \in F_{p}$ 에 대하여 $[\alpha]=[r \alpha]$ 을 만족하므로 $[\alpha]^{n}$ 의 계산을 $[r \alpha]^{n}$ 으로 바꾸어 계산할 수 있다, 따라서 주어진 메 시지가 $[\alpha]$ 라 할 때 $[\alpha]^{n}=[r \alpha]^{n}$ 으로 $[\alpha]$ 를 $[r \alpha]$ 로 랜덤화하여 계산할 수 있으므로 제1차 $\mathrm{DPA}\left(\mathrm{Di}^{-}\right.$ fferential Power Analysis)를 쉽게 막을 수 있다. 물론 이때는 [알고리즘 1]을 사용할 수 없으며 $F_{p}(\omega)$ 의 일반적인 지수 연산 알고리즘을 사용하게 된다.

\section{IV. 결론}

본 논문에서는 $\mathrm{TBC}$ 중에 가장 기본적인 이차확장 체에서의 상군 $T_{2}(p)$ 에 대한 연산방법과 부채널공격 에 안전한 효율적인 알고리즘을 제안하였다, 제안된 알고리즘은 SPA 공격을 막을 뿐만 아니라 지수 연산 도 더 효율적으로 수행할 수 있다는 장점이 있다. 또 한 상군의 특성상 지수 연산에서도 자연스럽게 메시지 를 난수화할 수 있으므로 제 1 차 DPA 공격에도 추가 적인 연산량 없이 효율적으로 방어할 수 있음을 알았 다. 본 연구에서 제안된 방법은 모든 $\mathrm{TBC}$ 에 자연스 럽게 확장할 수 있을 것으로 예상되며 따라서 $\mathrm{TBC}$ 은 키 크기의 감소뿐 만 아니라 부채널공격을 고려한 연 산의 효율성에서도 다른 암호에 비해 장점을 갖고 있 음을 알 수 있다.

\section{참고문헌}

[1] T. ElGamal, "A public key cryptosystem and a signature scheme based on discrete logarithms," IEEE Trans. on Information Theory, vol. 31, no 4, pp. 469-472, July 1985.

[2] FIPS PUB 186-3, “Digital Signature 
Standard (DSS)," Information Technology Laboratory, NIST. June 2009.

[3] C.P. Schnorr, "Efficient signature generation by smart cards," Journal of Cryptology, vol. 4, no. 3, pp. 161-174, Feb. 1991.

[4] P. Smith and C. Skinner, "A public-key cryptosystem and a digital signature system based on the Lucas function analogue to discrete logarithms," Adv. Crypto.- Asiacrypt'94, LNCS 917, pp. 355-364. Nov. 1994.

[5] A.K. Lenstra and E.R. Verheul, "The XTR public key system," Adv. Cryptol.CRYPTO 2000, LNCS 1880, pp. 1-19, Aug. 2000.

[6] 박영호, 오상호, 주학수, "공개 파라메터 키 크기를 줄인 새로운 이산대수문제," 한국정보보호학회논 문지, 13(2), pp. 91-98, 2003년 4월.

[7] K. Rubin and A. Silverberg, "Torus-based cryptography," Adv. Crypto.-CRYPTO, LNCS 2729, pp. 349 - 365, Aug. 2003.

[8] M. van Dijk and D. Woodruff, “Asymptotically optimal communication for torus-based cryptography," Adv. Cryptol.-CRYPTO, LNCS 3152, pp. $151-178$, Aug. 2004.

[9] M. van Dijk, R. Granger, D. Page, K. Rubin, A. Silverberg, M. Stam, and D. Woodruff, "Practical cryptography in high dimensional tori," Adv. Crypto.EUROCRYPT, LNCS 3494, pp. 234 - 250, May 2005.

〔10] K. Rubin and A. Silverberg, "Compression in finite fields and torus based cryptography," SIAM J. Comput., vol. 37. no 5. pp. 1401 -1428, Jan. 2008.

[11] D. Hankerson, A. Menezes, and S. Vanstone, Guide to Elliptic Curve
Cryptography. New York: Springer-Verlag, 2004.

〔12] R. Granger, D. Page, and M. Stam, "On small characteristic algebraic tori in pairing-based cryptography," LMS J. Comput. Math., vol. 9, pp.64-85, March 2006.

〔13] K. Karabina, "Torus-Based Compress ion by Factor 4 and 6," Trans. on Information Theory, vol 58, no 5, pp. 3293-3304, May 2012.

〔14] P Kocher, J Jaffe and B Jun, "Differential Power Analysis," Adv. Cryptol.CRYPTO, LNCS 1109, pp. 388 - 397, Aug. 1999.

〔15] J. Chung and A. Hasan, "Security Analysis of XTR Exponentiation Algorithms against Simple Power Analysis Attack," Preprint of CACR, Univ. of Waterloo, CACR 2004-05.

〔16] D. Page and M. Stam, "On XTR and Side-Channel Analysis," Selected Areas in Cryptography (SAC 2004), LNCS 3357 , pp. 54-68, Aug. 2004.

〔17] D.-G. Han, T. Izu, J. Lim, and K. Sakurai, "Side Channel Cryptanalysis on XTR Public Key Cryptosystem," IEICE Trans. Fund. S. S. on Disc. Math. and Its Applications, vol. E88-A, no. 5, pp.1214-1223, May 2005.

(18] M. Stam and A. K. Lenstra, "Efficient Subgroup Exponentiation in Quadratic and Sixth Degree Extensions," CHES 2002, LNCS 2523, pp. 318-332. Aug. 2002.

〔19] A.K. Lenstra and E.R. Verheul, "The XTR public key system," Advances in Cryptology-CRYPTO 2000, LNCS 1880 , pp. 1-19, Aug. 2000. 


\section{〈저 자 소 개〉}

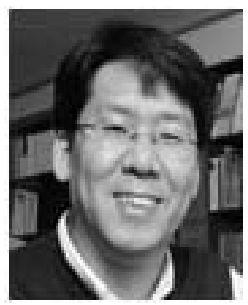

박 영 호 (Young-Ho Park) 종신회원 1990년 2월: 고려대학교 수학과 이학사 1993년 2월: 고려대학교 수학과 이학석사 1997년 2월: 고려대학교 수학과 이학박사 2002년 3월 현재: 세종 사이버 대학교 부교수

〈관심분야〉 정수론, 공개키 암호, 암호 프로토콜, 부채널 공격

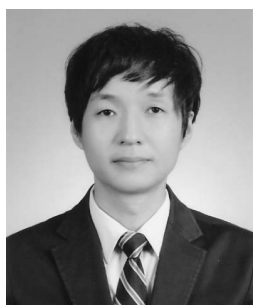

장 남 수 (Nam Su Chang) 종신회원

2002년 2월: 서울 시립대학교 수학과 이학사

2004년 8월: 고려대학교 정보보호 대학원 공학석사

2010년 2월: 고려대학교 정보경영공학전문대학원 공학박사

2010년 3월 2010년 6월 : 고려대학교 정보보호연구원 연구교수

2010년 7월 현재: 세종사이버대학교 조교수

〈관심분야〉 암호칩 설계 기술, 부채널 공격, 공개키 암호 알고리즘, 공개키 암호 암호분석 